ARTICULOS ORIGINALES Rev Chil Salud Pública 2013; Vol 17 (1): 35-39

Marcelo Godor ${ }^{14}$, José A. de Grazia', Álvaro Otárola ${ }^{2}$, Pamela Serón ${ }^{3}$.

'Médico Becado de Radiología, Hospital Clínico Universidad de Chile. ${ }^{2}$ Médico Radiólogo, Jefe Unidad de Imagenología, Clínica Portada de Antofagasta. ${ }^{3}$ Kinesióloga,

Magister en Epidemiología Clínica,

Universidad de la Frontera. ${ }^{a}$ Médico en Etapa de Destinación y Formación, Servicio de Salud Araucanía Sur.

Trabajo presentado en el Congreso Chileno de Radiología / Primer Encuentro Franco-Chileno de Radiología 2011.

Correspondencia a: Dr. Marcelo Godoy Z. Av. Santos Dumont 999, $1^{\text {er }}$ Piso Sector D. Santiago - Chile. Fono: 9788412 marzagod@gmail.com

\section{Caracterización de las propiedades diagnósticas de médicos generales del Servicio de Salud Araucanía Sur para el diagnóstico radiológico de neumonía adquirida en la comunidad}

\section{RESUMEN}

Objetivos: Caracterizar las propiedades diagnósticas de un grupo de médicos generales (MGs) del Servicio de Salud Araucanía Sur para el diagnóstico radiológico de neumonía adquirida en la comunidad (NAC).

Método: 201 pacientes adultos del Hospital de Gorbea fueron seleccionados según la presencia de cuadro clínico compatible con NAC. Se evaluó la sensibilidad, especificidad y valores predictivos de la interpretación de radiografía de tórax para el diagnóstico de NAC, obtenidas del consenso de $5 \mathrm{MGs}$, comparándolo con la interpretación radiográfica de médico radiólogo (gold standard).

Resultados: La evaluación de radiografías por MGs informó 89 casos con diagnóstico de NAC (44\%); en la evaluación realizada por radiólogo fueron 51 casos (25\%). El valor Kappa encontrado fue de 0,46 [0,34-0,57], considerado una concordancia moderada. La sensibilidad de los MGs para el diagnóstico radiológico de NAC fue de 86,5\% [74-94], la especificidad fue de 70,4\% [62-77]. El valor predictivo positivo fue de 50,6\% [39-61] y el valor predictivo negativo fue de 93,7\% [87-97].

Conclusiones: Los MGs presentan una buena sensibilidad para el diagnóstico radiológico de NAC; la especificidad es más baja, determinando un alto número de falsos positivos. La mayor utilidad del uso de radiografía de tórax por MGs para la evaluación de pacientes con sospecha de NAC, radica en su alta capacidad para detectar casos negativos, pero fallan en el $50 \%$ de los casos al afirmar ver una condensación.

Palabras clave: Radiografía de tórax, neumonía adquirida en la comunidad.

\section{Characterization of general PhysiCians' diagnostic abilities IN X-RAY OF COMMUNITY-ACQUIRED PNEUMONIA IN THE Araucania Sur Health Service}

\section{ABSTRACT}

Objective: Characterize the diagnostic abilities of a group of general physicians (GPs) in the Araucania Sur Health Service, for X-ray diagnosis of community-acquired pneumonia (CAP). 
Methods: 201 adult patients presenting a clinical profile suggesting CAP were selected at Gorbea Hospital. Sensitivity, specificity, and predictive variables for interpretation of chest $X$-rays for CAP diagnosis were evaluated. Diagnosis was obtained by a consensus of 5 GPs, and compared with the diagnosis of a radiologist (gold standard). Results: $X$-ray interpretation by GPs found 89 cases of CAP (44\%), while the radiologist's interpretation found 51 cases (25\%). The Kappa value found was 0.46 [0.34-0.57], which concludes moderate agreement. The sensitivity of GP X-ray interpretation was $86.5 \%$ [74-94], and specificity was $70.4 \%$ [62-77]. The positive predictive value was 50.6\% [39-61] and the negative predictive value was $93.7 \%$ [87-97].

Conclusions: The GPs have good sensitivity for $X$-ray diagnosis of $C A P$, while specificity was lower and included a high number of false positives. The usefulness of $X$-ray diagnosis by $G P$ with suspected CAP is determined by their ability to detect negative cases, which they failed to do in $50 \%$ of cases when they stated that they saw fogging on the $X$-ray.

Key words: Chest X-ray, community-acquired pneumonia

\section{INTRODUCCIÓN}

La prevalencia de neumonía adquirida en la comunidad (NAC) en servicios de atención ambulatoria (consultorios y servicios de urgencia) alcanza entre $3 \%$ a $5 \%$ de las consultas por síntomas respiratorios ${ }^{1}$.

$\mathrm{La}$ anamnesis y examen físico carecen de sensibilidad y especificidad para establecer el diagnóstico clínico de NAC, por lo tanto, no permiten diferenciarla de otras condiciones respiratorias agudas en muchos $\operatorname{casos}^{1,2}$. Debido a lo anterior, la Sociedad Americana de Tórax y la Sociedad Americana de Enfermedades Infecciosas recomiendan solicitar radiografía de tórax a todos los pacientes con sospecha clínica de NAC ${ }^{3,4}$. La Sociedad Chilena de Enfermedades Respiratorias también sugiere confirmar el diagnóstico de NAC con una radiografía de tórax ${ }^{1}$.

La radiografía de tórax es un examen ampliamente disponible en el sistema público de salud de nuestro país, debiendo ser interpretados los hallazgos radiológicos en muchos casos por médicos generales (MGs). En este contexto, cobra importancia la experiencia del operador para lograr un adecuado diagnóstico radiológico.

La literatura internacional describe que existe una buena concordancia interobservador a la hora de identificar la presencia o ausencia de infiltrado $^{5,6}$. Sin embargo, hay una alta variabilidad interobservador cuando se trata de definir el tipo de infiltrado o la presencia de broncograma aé- reo $^{6}$. El diagnóstico radiológico de NAC es dificultoso y la experiencia del observador es importante ${ }^{7,8}$.

No se dispone de estudios nacionales que evalúen la concordancia interobservador, ni las propiedades diagnósticas (sensibilidad, especificidad y valores predictivos) de MGs para el diagnóstico radiológico de NAC.

\section{OBJETIVOS}

Caracterizar las propiedades diagnósticas de un grupo de MGs del Servicio de Salud Araucanía Sur para el diagnóstico radiológico de NAC, comparándolo con la interpretación radiológica por médico radiólogo (gold standard). Además, calcular la concordancia interobservador para este mismo examen entre MGs y médico radiólogo.

\section{MÉTODO}

El cálculo de tamaño de la muestra fue estimado según un estudio local previo?: con prevalencia de $40 \%$, sensibilidad y especificidad de $95 \%$ y $35 \%$ respectivamente, $9 \%$ de error y $95 \%$ de confianza, el tamaño muestral calculado fue de 200 pacientes. Además, en base a lo publicado en la literatura, que establece un coeficiente Kappa de 0,43 entre médicos radiólogos y médicos residentes de radiología ${ }^{8}$, se estimó con una prevalen- 
cia de $40 \%$ (según estudio piloto) ${ }^{9}$ y un $95 \%$ de confianza, una precisión de 0,15 para la estimación de concordancia ${ }^{8-10}$.

Se incluyeron en el estudio 201 pacientes adultos consultantes por cuadro agudo respiratorio (tos, coriza, odinofagia, disnea, fiebre y/o compromiso del estado general), que además presentaron anomalías al examen físico pulmonar, provenientes del Servicio de Urgencia y del Policlínico del Hospital de Gorbea. Se excluyó del estudio a pacientes con hospitalización y/o uso de antibiótico el mes previo, además de pacientes con anomalías conocidas en la radiografía de tórax. A los pacientes se les solicitó una radiografía de tórax (proyección posteroanterior y lateral), para confirmar o descartar el diagnóstico de NAC. El tiempo desde la consulta a la toma de la radiografía no excedió los 3 días.

Las placas radiográficas fueron evaluadas en conjunto por grupos de $2 \mathrm{MGs}$ (de un total de 5), llegando éstos a un consenso sobre la presencia o ausencia de infiltrado compatible con NAC. Se consideró caso positivo a aquellos que demostraron imagen de condensación, definida como opacidad pulmonar, con o sin broncograma aéreo ${ }^{11}$. Posteriormente, un médico radiólogo revisó independientemente estas placas, llegando a su propia conclusión y evaluando la calidad técnica de las mismas.

Ocupando un modelo de test diagnóstico, en donde la evaluación por médico radiólogo fue considerada como gold standard, se calcularon los intervalos de confianza (95\%) para los estimadores de propiedades diagnósticas de sensibilidad y especificidad, así como también el valor predictivo positivo y negativo. También se calculó, con 95\% confianza, el coeficiente Kappa como medida de concordancia entre MGs y médico radiólogo. Se utilizó el software estadístico Stata 9.0 para la realización de los cálculos.

Se hizo además seguimiento clínico de los pacientes, hasta completado el tratamiento antibiótico, para observar la evolución de acuerdo al diagnóstico y evaluar posibles diagnósticos diferenciales.

El protocolo de estudio fue aprobado por el Comité de Ética del Servicio de Salud Araucanía Sur.

\section{RESULTADOS}

La edad promedio de la muestra fue 68 años $(\mathrm{DE} \pm 21)$. Existió leve predominio femenino (53\%). El $90 \%$ de los pacientes provenía del Servicio de Urgencia y el 10\% restante del Policlínico. Respecto a patologías crónicas, el 52\% de la muestra presentaba hipertensión arterial y el $18 \%$ diabetes mellitus tipo 2. En relación al motivo de consulta, los principales fueron tos (39\%), fiebre (28\%) y compromiso del estado general (24\%).

Del total de radiografías evaluadas, los MGs realizaron diagnóstico radiológico de NAC en 89 casos, lo que corresponde a una prevalencia de $44 \%$. El médico radiólogo realizó diagnóstico radiológico de NAC en 51 casos, correspondiente (prevalencia de 25\%). Con estos valores, la concordancia calculada entre MGs y médico radiólogo da un coeficiente Kappa de 0,46 [0,34-0,57], calificado como concordancia moderada ${ }^{10}$.

En relación a las propiedades diagnósticas de los MGs al interpretar una radiografía de tórax en busca de NAC, los valores obtenidos fueron los siguientes: sensibilidad 86,5\% [74-94], especificidad: $70,4 \%$ [62-77], valor predictivo positivo: 50,6\% [39-61] y valor predictivo negativo: 93,7\% [87-97]. La información se resume en la Tabla 1.

De los 89 pacientes diagnosticados por los MGs como NAC, hubo 44 casos correspondientes a falsos positivos. De éstos, 26 correspondían a placas sin hallazgos patológicos en la evaluación por médico radiólogo. En los 18 casos restantes se obtuvo un diagnóstico alternativo en la evaluación por médico radiólogo, siendo los más frecuentes el edema pulmonar agudo (10 casos) y las secuelas de TBC (4 casos). La información se resume en la Tabla 2.

En el seguimiento clínico de los pacientes no se pesquisaron otros diagnósticos alternativos. Hubo 10 pacientes que fallecieron durante este período (letalidad de 5\%).

\section{DISCUSIÓN}

En la atención primaria, la interpretación radiológica certera es crítica para el manejo de los casos de NAC, pues una radiografía positiva va a 
Tabla 1. Propiedades diagnósticas de médicos generales para el diagnóstico radiológico de NAC

\begin{tabular}{|c|c|c|c|}
\hline \multirow[t]{2}{*}{ Evaluación por MGs } & \multicolumn{2}{|c|}{ Evaluación por médico radiólogo } & \multirow[t]{2}{*}{ Total } \\
\hline & Condensación & No condensación & \\
\hline Condensación & 45 (VP) & $44(\mathrm{FP})$ & 89 \\
\hline No condensación & $7(\mathrm{FN})$ & $105(\mathrm{VN}) \mathrm{x}$ & 112 \\
\hline Total & 52 & 149 & 201 \\
\hline
\end{tabular}

Sensibilidad (S): $86,5 \%$

Especificidad (E): 70,4\%
Valor predictivo positivo (VPP): $50,6 \%$

Valor predictivo negativo (VPN): 93,7\%

Tabla 2. Diagnóstico radiológico de los falsos positivos evaluados por médico radiólogo

\begin{tabular}{|lc|}
\hline Diagnóstico según médico radiólogo & Número de casos \\
\hline Radiografía normal & 26 \\
Edema pulmonar agudo & 10 \\
Secuelas de TBC (cicatrices pulmonares) & 4 \\
Infiltrado intersticial aislado, sin condensación & 3 \\
Adenopatía hiliar & 1 \\
Total & 44 \\
\hline
\end{tabular}

determinar el inicio o la continuación de tratamiento antibiótico. En este contexto, cobra importancia la experiencia de quien interpreta las radiografías de tórax. La literatura internacional avala la dependencia de la experiencia del operador en el diagnóstico radiológico de $\mathrm{NAC}^{7,8}$. Nuestro estudio también lo ratifica, al demostrar un valor de coeficiente Kappa de 0,46, correspondiente sólo a una concordancia moderada entre MGs y médico radiólogo ${ }^{10}$.

Al evaluar las propiedades diagnósticas de los MGs para el diagnóstico radiológico de NAC, se obtiene una buena sensibilidad $(86,5 \%)$ y una especificidad moderada (70,4\%); esto último, determina un alto número de falsos positivos. Analizando los valores predictivos, el alto valor predictivo negativo $(93,7 \%)$ pone en evidencia que la mayor utilidad del uso de radiografía de tórax por MGs para la evaluación de pacientes con sospecha de NAC radica en la alta capacidad para detectar casos negativos; por el contrario el bajo valor predictivo positivo obtenido $(50,6 \%)$ indica que los MGs fallan casi en el 50\% de los casos al afirmar ver una condensación. Al modelar los valores predictivos obtenidos, según el teorema de Bayes, bajando la prevalencia de NAC a 5\% (de acuerdo a la literatura nacional) ${ }^{1}$ el valor predictivo negativo asciende a $99,0 \%$ y el valor predictivo positivo desciende a $13,3 \%$. Lo anterior respalda aún más que el principal rol de los MGs al evaluar radiografías de tórax de pacientes con sospecha de NAC es identificar aquellos casos con radiografía normal. No se dispone de estudios nacionales que permitan comparar nuestros resultados con los existentes en otras zonas del país.

En relación a los 44 casos falsos positivos, llama la atención que la mayoría de éstos (26) correspondían a radiografías normales, pesquisándose diagnósticos alternativos por el médico radiólogo sólo en 18 casos. Lo anterior pone en evidencia que los MGs, a pesar de ser buenos en identificar radiografías de tórax normal, presentan algunas dificultades al interpretar la anatomía radiográfica normal del tórax.

Los resultados de nuestro estudio resaltan la necesidad de contar con una mejor formación 
radiológica de los MGs, ya sea con la incorporación de rotaciones por servicios de radiología en la malla curricular de pregrado, o bien mediante cursos de capacitación durante sus años de estadía como MGs.

Como limitaciones y posibles fuentes de sesgo, cabe mencionar que nuestro estudio describe la realidad local de un hospital pequeño, lo que limita la generalización de nuestros resultados a la realidad nacional; se requiere de estudios de mayor tamaño, multicéntricos, para aumentar la validez externa. Otro aspecto, radica en la selección de los pacientes, pues se seleccionó pacientes con síntomas respiratorios y alteraciones al examen físico pulmonar, lo cual entrega una prevalencia de NAC de alrededor de $25 \%$, valor significativamente mayor al $5 \%$ descrito en consultantes por síntomas respiratorios en la literatura nacional ${ }^{1}$. Por último, la validez interna del estudio se ve limitada por el hecho de que son los mismos MGs los que muchas veces evaluaron clínicamente al paciente y luego realizaron la interpretación radiográfica; lo anterior genera sesgo al aumentar la sensibilidad y disminuir la especificidad del diagnóstico radiológico de los MGs, esto se intentó disminuir al establecer evaluación radiológica por grupos de 2 MGs.

\section{CONCLUSIONES}

Los MGs del Servicio de Salud Araucanía Sur presentan una buena sensibilidad para el diagnóstico radiológico de NAC; no ocurre así con la especificidad, donde el valor es más bajo, determinando un alto número de falsos positivos. La mayor utilidad del uso de radiografía de tórax por MGs para la evaluación de pacientes con sospecha de NAC, radica en su alta capacidad para detectar casos negativos, pero fallan en casi el $50 \%$ de los casos al afirmar ver una condensación.

\section{BIBLIOGRAFÍA}

1. Gil R, Fernández P, Sabbagh E. Diagnóstico clínico-radiológico de la neumonía del adulto adquirida en la comunidad. Rev Chil Infect 2005; 22(Supl 1): S26-S31.
2. Metlay JP, Kapoor WN, Fine MJ. Does this patient have community-acquired pneumonia? Diagnosing pneumonia by history and physical examination. JAMA 1997; 278: 1440-5.

3. Niederman MS, Mandell LA, Anzueto A, Bass JB, Broughton WA, Campbell GD, et al. Guidelines for the management of adults with community-acquired pneumonia. Diagnosis, assessment of severity, antimicrobial therapy, and prevention. Am J Respir Crit Care Med 2001; 163: 1730-54.

4. Bartlett JG, Dowell SF, Mandell LA, File Jr TM, Musher DM, Fine MJ. Practice guidelines for the management of community-acquired pneumonia in adults. Infectious Diseases Society of America. Clin Infect Dis 2000; 31: 347-82.

5. Young M, Marrie TJ. Interobserver variability in the interpretation of chest roentgenograms of patients with possible pneumonia. Arch Intern Med 1994; 154(23): 2729-32.

6. Albaum MN, Hill LC, Murphy M, Li YH, Fuhrman CR, Britton CA, et al. Interobserver reliability of the chest radiograph in communityacquired pneumonia. PORT Investigators. Chest 1996; 110(2): 343-50.

7. Melbye $\mathrm{H}$, Dale K. Interobserver variability in the radiographic diagnosis of adult outpatient pneumonia. Acta Radiol 1992; 33(1): 79-81.

8. Ojutiku O, Haramati LB, Rakoff S, Sprayregen $S$. Radiology residents' on-call interpretation of chest radiographs for pneumonia. Acad Radiol 2005; 12: 658-64.

9. Godoy M, Enríquez M, Loyola M. Uso del juicio clínico para el diagnóstico de neumonía comunitaria en adultos en hospital familiar y comunitario de Gorbea. Libro de Resúmenes, Congreso Médicos Generales de Zona. 2009. Pucón, Chile.

10. Kundel HL, Polansky M. Measurement of observer agreement. Radiology 2003; 228: 303-8.

11. Hansell DM, Dee P. Infections of the lungs and pleura. In: Armstrong P, Wilson A, Dee P, Hansell DM, editors. Imaging of diseases of the chest. $3^{\text {rd }}$ Ed. Mosby; 2000. Chapter 5. 